\title{
Composição química e atividade biológica dos óleos essenciais das folhas e caules de Rhodostemonodaphne parvifolia Madriñán (Lauraceae)
}

Joelma Moreira ALCÂNTARA ${ }^{1}$, Klenicy Kazumy de Lima YAMAGUCHI ${ }^{2}$, Jefferson Rocha de Andrade SILVA ${ }^{3}$ e Valdir Florêncio da VEIGA JUNIOR ${ }^{4}$

\section{RESUMO}

Os óleos essenciais das folhas e caules de Rhodostemonodaphne parvifolia coletada na Reserva Florestal Adolpho Ducke foram obtidos por hidrodestilaçáo e analisados por cromatografia em fase gasosa com detector de ionizaçáo de chama e espectrometria de massas. Treze constituintes foram identificados nas folhas, dos quais o $\beta$-cariofileno foi o que apresentou maior percentual (41,30\%). Nos caules foram identificados quinze compostos, com predominância dos sesquiterpenos $\beta$-cariofileno $(16,20 \%)$ e epi-cedrol $(13,30 \%)$ e do monoterpeno linalol $(15,40 \%)$. Os óleos foram analisados em ensaios químicos, de sequestro do radical livre DPPH, e enzimáticos, de inibição da enzima acetilcolinesterase. Nos ensaios de sequestro de radicais livres, apesar de terem se mostrado ativos em ensaios qualitativos, os óleos essenciais apresentaram fraca atividade antioxidante no ensaio quantitativo (inativos em concentração inferior a $1.000 \mu \mathrm{g} / \mathrm{mL}$ ). A inibição da acetilcolinesterase só foi observada nos óleos essenciais das folhas. A composição química e a atividade biológica destes óleos estão sendo relatadas pela primeira vez neste trabalho.

PALAVRAS-CHAVE: Rhodostemonodaphne parvifolia, óleo essencial, antioxidante, anticolinesterase.

\section{Composition and biology activity of essential oils from leaves and stems of Rhodostemonodaphne parvifolia Madriñán (Lauraceae)}

\section{ABSTRACT}

The essential oils of leaves and stems of Rhodostemonodaphne parvifolia collected in the Adolpho Ducke Forest Reserve were obtained by hydrodistillation and analyzed by GC-FID and GC-MS. Thirteen components were identified in the leaf oil, of which $\beta$-caryophyllene was the major (41.30\%). Fifty components were identified in the stem oil, among them the sesquiterpenes $\beta$-caryophyllene (16.20\%) and epi-cedrol (13.30\%), together with the monoterpene linalool (15.40\%). The essential oils were analyzed at chemical, DPPH free radical scavenging; and enzymatic bioassays, acetylcholinesterase inhibition. At antioxidant $\mathrm{DPPH}$ assays, the oils showed to be active at the qualitative assay but only weakly active at quantitative test (inactive at concentration lower than $1,000 \mu \mathrm{g} / \mathrm{mL}$ ). The inhibition of acetylcholinesterase was detected only in leaves essential oils. This paper describes for the first time the composition of these essential oils and their biological activities.

KEY WORDS: Rhodostemonodaphne parvifolia, essential oil, antioxidant, anticholinesterase.

\footnotetext{
1 Universidade Federal do Amazonas.E-mail: valdirveiga@ufam.edu.br

2 Universidade Federal do Amazonas.E-mail: jomalc@yahoo.com.br

3 Universidade Federal do Amazonas.E-mail: klenicy@yahoo.com.br

${ }^{4}$ Universidade Federal do Amazonas.E-mail: jrocha_01@ufam.edu.br
} 


\section{INTRODUÇÃO}

Os óleos essenciais são capazes de proteger os sistemas biológicos devido a sua atividade antiradical livre contra radicais alquila, e em menor grau, contra o ânion superóxido (Pascual et al., 1994). Este efeito é observado especialmente nas membranas lipídicas, protegendo-as dos danos produzidos pelo estresse oxidativo, considerado a principal causa do envelhecimento, das doenças cardiovasculares, neurodegenerativas e das células tumorais (Cozzi et al., 1997; Gülcin et al, 2003; Atoui et al., 2005; Sacchetti et al., 2005).

Entre as doenças neurodegerativas, o Mal de Alzheimer atinge inicialmente a memória e em seguida a capacidade de raciocínio e a comunicação. Os sintomas dessa doença estão associados à redução de neurotransmissores cerebrais, como acetilcolina, noradrenalina e serotonina (Bryne, 1998). O tratamento para o Mal de Alzheimer é sintomático e consiste basicamente na tentativa de restauração da função colinérgica, sendo amplamente utilizados para este fim os inibidores de acetilcolinesterase (Perry, 1986). Os medicamentos disponíveis no mercado são relativamente caros, o que torna a busca de novos inibidores de origem vegetal uma alternativa interessante, na medida em que estes seriam economicamente mais viáveis.

Nesse contexto, a pesquisa por novos óleos essenciais, nunca antes descritos, especialmente da Amazônia, tem particular interesse na busca por substâncias inibidoras de acetilcolinesterases e de radicais livres. A família Lauraceae é uma das mais importantes produtoras de óleos, com elevado endemismo na Amazônia e com grande potencial comercial. Rhodostemonodaphne Rohwer \& kubitzki é um gênero botânico pertencente à família Lauraceae, com cerca de 40 espécies distribuídas na América do Sul (Madriñán, 2004) e com pouquíssimos estudos já realizados, tendo sido incluída na lista de espécies da flora brasileira com deficiência de dados (MMA, 2008).

No presente estudo, é feita a descrição inédita da composição química e a avaliação do potencial antioxidante pelo ensaio de captura de radicais livres DPPH e a avaliaçáo do potencial de inibiçấo da enzima acetilcolinesterase dos óleos essenciais das folhas e dos caules de Rhodostemonodaphne parvifolia Madriñán.

\section{MATERIAL E MÉTODOS}

\section{MATERIAL VEGETAL}

As amostras de Rhodostemonodaphne parvifolia Madriñán foram coletadas na Reserva Florestal Adolpho Ducke, Manaus, Amazonas, nos meses de março de 2007 e 2008. Todo o material vegetal foi obtido de um mesmo indivíduo, na fase estéril. A espécie tem exsicata depositada e foi identificada no Projeto Flora da Reserva Ducke (Ribeiro et al., 1999).

\section{EXTRAÇÃO DOS ÓLEOS ESSENCIAIS}

Amostras, recém coletadas, de folhas e caules foram secas à sombra, reduzidas em moinho de facas e submetidas, separadamente, à hidrodestilação por um período de 4 h, utilizando aparelho do tipo Clevenger modificado. Os óleos essenciais extraídos foram secos com sulfato de sódio anidro, acondicionados em pequenos frascos de vidro âmbar e mantidos sob refrigeração. $\mathrm{O}$ cálculo do rendimento foi realizado através da relação da massa do óleo obtido com a massa de material vegetal utilizada na extração.

\section{IDENTIFICAÇÃO DOS CONSTITUINTES DOS ÓLEOS ESSENCIAIS}

Os óleos essenciais foram diluídos em hexano $(5 \mathrm{mg} /$ $\mathrm{mL}$ ) e as soluçóes obtidas foram submetidas à análise por cromatografia em fase gasosa acoplada a detector de ionização de chama (CG-DIC), para a análise quantitativa de seus constituintes e obtenção dos índices de retenção e, posteriormente, à análise por cromatografia em fase gasosa acoplada a detector de espectrometria de massas (CG-EM), para a análise qualitativa.

Os índices de retenção foram calculados em relação aos tempos de eluição das substâncias e dos tempos de eluição de uma série de hidrocarbonetos lineares $\left(\mathrm{C}_{9}-\mathrm{C}_{22}\right)$ que foram co-injetada com a amostra em CG-DIC. A identificação dos constituintes foi obtida com o conjunto de dados de índices de retenção e dos espectros de massas, comparando com dados da literatura (Adams, 2001) e de espectroteca Wiley 7.0.

Os óleos foram analisados em cromatógrafo em fase gasosa modelo CG 2010 da Shimadzu ${ }^{\odot}$ com detector por ionização de chama (CG-DIC). As análises foram realizadas com coluna CP-Sil 5 CB (100\% dimetilpolisiloxano) da $\operatorname{Varian}^{\oplus}$, com medidas de $15 \mathrm{~m} \times 0,25 \mathrm{~mm} \times 0,25 \mu \mathrm{m}$, sendo utilizado hélio como gás de arraste em um fluxo de $2,0 \mathrm{~mL} / \mathrm{min}$. A injeçáo em modo split 1:10 foi realizada com injetor a $250^{\circ} \mathrm{C}$. A temperatura do detector foi de $290^{\circ} \mathrm{C}$ e o forno foi programado de $60^{\circ} \mathrm{C}$ a $180^{\circ} \mathrm{C}$ a $3{ }^{\circ} \mathrm{C} / \mathrm{min}$. Foram co-injetados padróes de hidrocarbonetos lineares para a determinação dos índices de retenção. Após a análise por CG-DIC, os óleos foram analisados em cromatógrafo em fase gasosa modelo QP-2010 da Shimadzu ${ }^{\odot}$ com detector por espectrometria de massas (CG-EM). As análises foram realizadas com coluna VF-1MS da Varian ${ }^{\oplus}$, com medidas de $15 \mathrm{~m} \times 0,25 \mathrm{~mm} \times 0,25 \mu \mathrm{m}$. As condiçóes de análise do forno foram as mesmas utilizadas por CG-DIC, utilizando a técnica de impacto eletrônico a $70 \mathrm{eV}$. 


\section{ANÁLISE QUALITATIVA DA ATIVIDADE ANTIOXIDANTE}

Os óleos essenciais obtidos de $R$. parvifolia foram avaliados com relação a sua capacidade de captura de radical livre estável 2,2'-difenil-1-picrilhidrazila (DPPH), por Cromatografia em Camada Delgada (CCD) segundo método de Blois (1958). Foram aplicados $10 \mu \mathrm{L}$ de cada óleo e da substância de referência (quercetina dissolvida em metanol $(1 \mathrm{mg} / \mathrm{mL})$ ) sobre a cromatoplaca.

Após a evaporação do solvente a placa foi nebulizada com a soluçáo de DPPH. Depois de trinta minutos foi realizada a leitura da placa, em que halos amarelados sobre fundo de coloração púrpura, são considerados positivos.

\section{ANÁLISE QUANTITATIVA DA ATIVIDADE ANTIOXIDANTE}

A atividade antioxidante foi avaliada por meio da capacidade sequestrante do DPPH, utilizando também quercetina como padrão. As soluçôes foram preparadas em metanol, para cada óleo analisado, com concentraçóes de 5 a $250 \mu \mathrm{g} / \mathrm{mL}$. O mesmo procedimento foi realizado para a soluçáo padráo com concentraçáo de $0,3125 \mathrm{a} 5 \mu \mathrm{g} / \mathrm{mL}$.

As leituras da absorbância da amostra foram realizadas após 30 minutos da adição da solução de DPPH $(0,3 \mathrm{mmol} / \mathrm{L})$ em espectrofotômetro UV-Vis (modelo Biomat 3 da Thermo Electron Corporation) no comprimento de onda $517 \mathrm{~nm}$, em cubeta de quartzo. Através dos dados obtidos e por regressão linear foram obtidos os valores de concentração eficiente $\left(\mathrm{CE}_{50}\right)$ (Mensor, 2001).

\section{MEDIDA DA ATIVIDADE INIBIDORA DE ACETILCOLINESTERASE}

$\mathrm{O}$ ensaios de inibição da enzima acetilcolinesterase (AChE) foi avaliada seguindo a metodologia descrita por Elmann et al. (1961), adaptada por Rhee et al. (2001). Este bioensaio consiste na aplicação da amostra em CCD, seguida da pulverização da placa com o reagente de Ellman (ácido 5,5'-ditiobis-[2-nitrobenzóico, DTNB) e uma solução de iodeto de acetiltiocolina (ATCI) em tampão e deixa secar. Após este procedimento, pulveriza-se a placa com a enzima $\operatorname{AChE}(3 \mathrm{U} / \mathrm{mL})$. Decorridos alguns minutos $(-10)$, a inibiçáo enzimática pode ser constatada pela ausência da cor amarela e concomitante surgimento de um halo branco. Como padráo positivo de atividade foi utilizada eserina.

\section{RESULTADOS E DISCUSSÃO}

A relação dos constituintes químicos dos óleos essenciais obtidos das folhas e caules de $R$. parvifolia, rendimentos, suas quantidades relativas e respectivos índices de retenção (IR) são apresentados na Tabela 1.

Através dos dados espectrométricos e de cromatografia foram detectados 20 compostos presentes nos dois óleos analisados. O percentual da composição química dos óleos essenciais determinado foi de $100 \%$ e o rendimento obtido foi relativamente muito baixo.

Dos constituintes químicos detectados no óleo essencial das folhas $14,13 \%$ são monoterpenos e $85,87 \%$ são sesquiterpenos, em que os constituintes majoritários foram o $\beta$-cariofileno (41,30\%), $\beta$-selineno (13,60\%) e B germacreno $(7,75 \%)$. No óleo essencial dos caules também se observou teores menores de monoterpenos $(15,40 \%)$ em relaçáo aos sesquiterpenos $(84,60 \%)$, tendo como majoritários $\beta$-cariofileno $(16,20 \%)$, linalol $(15,40 \%)$ e epi-cedrol $(13,30 \%)$.

O perfil químico desses óleos revelou uma proporção elevada de sesquiterpenos, principalmente de hidrocarbonetos sesquiterpênicos. Na maioria dos óleos essenciais de Lauraceae reportados na literatura observa-se a predominância de sesquiterpenos, principalmente de hidrocarbonetos sesquiterpênicos (Gottlieb et al., 1981; Cicció \& Chaverri, 2008; Telascrea et al., 2008).

O metabólito mais abundante e comum entre os óleos essenciais analisados foi o $\beta$-cariofileno. Este sesquiterpeno é descrito em diversos óleos essenciais por possuir forte aroma e diversas atividades biológicas, tais como: anti-inflamatória (Fernandes et al., 2007; Passos et al., 2007), antialérgica (Ghelardini et al., 2001), anestésica local (Costa et al., 2000), antifúngica (Zheng et al., 1992) e anticarcinogênica (Chinou et al., 1996).

A presença de hidrocarbonetos monoterpênicos só foi observada no óleo essencial das folhas sendo representada apenas por duas substâncias com porcentagem inferior a 1\% ( $\alpha$-pineno e sabineno).

O linalol foi o único monoterpeno oxigenado encontrado no óleo essencial dos caules, além de ser um dos seus constituintes majoritários. Nas folhas, além do linalol $(5,71 \%)$ detectou-se a presença de isovalerato de linalol $(6,77 \%)$.

Os resultados dos bioensaios das atividades antioxidantes qualitativa e quantitativa e inibidora de acetilcolinesterase encontram-se descritos na Tabela 2.

$\mathrm{O}$ ensaio de atividade antioxidante frente ao radical DPPH em placas cromatográficas de gel de sílica revelou atividade de captura de radicais livres, porém no ensaio quantitativo os óleos só apresentaram atividade nas concentraçóes acima de $1000 \mu \mathrm{g} / \mathrm{mL}$.

Recentemente, foi comprovado que monoterpenos possuem atividades provedora de termotolerância, fotoproteção e antioxidante devido à capacidade de captarem radicais de oxigênio oriundos do processo fotossintético (Peńuelas \& Llusià, 2002; Peñuelas \& Munné-Bosch, 2005). 
Tabela 1 - Rendimentos e composição percentual dos óleos essenciais de Rhodostemonodapne parvifolia.

\begin{tabular}{|c|c|c|c|}
\hline \multirow{2}{*}{ Substância } & \multirow{2}{*}{$\mathbb{R}$} & \multicolumn{2}{|c|}{ R. parvifolia } \\
\hline & & Folhas & Caules \\
\hline 1. $\alpha$-pineno & 932 & 0,70 & - \\
\hline 2. sabineno & 968 & 0,95 & - \\
\hline 3. linalol & 1100 & 5,71 & 15,40 \\
\hline 4. $\delta$-elemeno & 1336 & - & 3,50 \\
\hline 5. $\alpha$-ylangeno & 1365 & 6,75 & - \\
\hline 6. $\alpha$-copaeno & 1376 & - & 2,46 \\
\hline 7. $\beta$-elemeno & 1388 & - & 2,43 \\
\hline 8. $\beta$-cariofileno & 1408 & 41,30 & 16,20 \\
\hline 9. E- $\alpha$-bergamoteno & 1434 & - & 1,92 \\
\hline 10. $\alpha$-humuleno & 1451 & 5,25 & 2,05 \\
\hline 11. isovalerato de linalol & 1469 & 6,77 & - \\
\hline 12. $\alpha$-amorfeno & 1476 & 3,53 & 3,65 \\
\hline 13. D germacreno & 1478 & - & 9,08 \\
\hline 14. $\beta$-selineno & 1484 & 13,60 & - \\
\hline 15. $\gamma$-cadineno & 1509 & 1,53 & 1,73 \\
\hline 16. elemol & 1544 & - & 5,81 \\
\hline 17. B germacreno & 1554 & 7,75 & 5,68 \\
\hline 18. santalenona & 1566 & 5,51 & 8,60 \\
\hline 19. epi-cedrol & 1614 & - & 13,30 \\
\hline 20. epi- $\alpha$-cadinol & 1637 & 0,65 & 8,19 \\
\hline Rendimento do óleo (\%) & & 0,07 & 0,01 \\
\hline Total de Hidrocarbonetos monoterpênicos & & 1,65 & - \\
\hline Total de Monoterpenos oxigenados & & 12,48 & 15,40 \\
\hline Total de Hidrocarbonetos sesquiterpênicos & & 79,71 & 48,70 \\
\hline Total de Sesquiterpenos oxigenados & & 6,16 & 35,90 \\
\hline
\end{tabular}

$\mathrm{IR}=$ Indice de Retenção.

Tabela 2 - Atividades antioxidante e inibidora de acetilcolinesterase dos óleos essenciais de Rhodostemonodapne parvifolia.

\begin{tabular}{|c|c|c|c|}
\hline & \multicolumn{2}{|c|}{ R. parvifolia } & \multirow{2}{*}{ Quercetina } \\
\hline & Folhas & Caules & \\
\hline Antioxidante qualitativo & + & + & + \\
\hline Antioxidante quantitativo $(\mu \mathrm{g} / \mathrm{mL})$ & $>1000$ & $>1000$ & 3,125 \\
\hline Inibição de acetilcolinesterase & + & - & - \\
\hline
\end{tabular}

Muitos mono- e sesquiterpenos tiveram sua atividade inibidora de acetilcolinesterase comprovada, tais como o elemol, linalol e $\alpha$-pineno (Miyazama et al., 1998; Perry et al., 2000), substâncias que também estão presentes no óleo essencial de $R$. parvifolia. Porém, quando estas substâncias são testadas em conjunto, a atividade inibidora da enzima pode ser reduzida ou intensificada devido ao efeito sinérgico e a inibição competitiva entre elas.

Através deste resultado podemos observar que os terpenos também possuem atividade inibidora de acetilcolinesterase, porém não se pode inferir se alguma substância isoladamente ou em conjunto com outras são as responsáveis por tal atividade.

\section{AGRADECIMENTOS}

Os autores agradecem à FAPEAM, CAPES e CNPq pelo apoio financeiro e ao CBA pelo apoio técnico nas análises cromatográficas.

\section{BIBLIOGRAFIA CITADA}

Adams, R.P. 2001. Identification of essential oil components by gas chromatography/ quadrupole mass spectroscopy, Allured, Illinois, $455 \mathrm{pp}$.

Atoui, A.K.; Mansouri, A.; Boskou, G.; Kefalas, P. 2005. Tea and herbal infusions: their antioxidant activity and phenolic profile. Food Chemistry, 89 (1): 999-1003.

Blois, M.S. 1958. Antioxidant determinations by the use of a stable free radical. Nature, 181: 1199-1200.

Bryne, G.J.A. 1998. Treatment of cognitive impairment in Alzheimer's disease. Australian Journal Hospital Pharmacy, 28: 261-266.

Chinou, I.B. ; Roussis, V. ; Perdetzoglou, D.; Loukis, A. 1996. Chemical and biological studies on two Helichrysum species of Greek origin. Planta Medica, 62 (4): 377-379. 
Cicció, J.F.; Chaverri, C. 2008. Volatile constituents of the oils from Povedadaphne quadriporata (Lauraceae) from "Alberto M. Brenes" biological preserve, Costa Rica. Química Nova, 31(3): 605-609.

Costa, T.R.; Fernandes, O.F.L.; Santos, S.C.; Oliveira, C.M.A.; Lião, L.M.; Ferri, P.H.; Paula, J.R.; Ferreira, H.D.; Sales, B.H.N.; Silva, M.R.R. 2000. Antifungal activity of volatile constituents of Eugenia dysenterica leaf oil. Journal of Ethnopharmacology, 72: 111-117.

Cozzi, R.; Ricordy, R.; Aglitti, T.; Gatta, V.; Perticone, P.; De Salvia, R. 1997. Ascorbic acid and b-carotene as modulators of oxidative damage. Carcinogenesis, 18: 223-228.

Ellman, G.L.; Courtney, K.D.; Andres, V.; Featherstone, R.M. 1961. A new and rapid colorimetric determination of acetylcholinesterase activity. Biochemical Pharmacology, 7: 88-95.

Fernandes, E.S.; Passos, G.F.; Medeiros, R.; da Cunha, F.M.; Ferreira, J.; Campos, M.M.; Pianowski, L.F.; Calixto, J.B. 2007. Anti-inflammatory effects of compounds alpha-humulene and (-)-trans-caryophyllene isolated from the essential oil of Cordia verbenacea. European Journal of Pharmacology, 569(3): 228-236.

Ghelardini, C.; Galeotti, N.; Di Cesare Mannelli, L.; Mazzanti, G.; Bartolini, A. 2001. Local anaesthetic activity of betacaryophyllene. II Farmaco, 56: 387-389.

Gottlieb, O.R.; Koketzu, M.; Magalhães, M.T.; Maia, J.G.S.; Mendes, P.H.; Rocha, A. I.; Silva, M.L.; Wilberg, V.C. 1981. Amazonian Essential Oils VII. Acta Amazonica, 11(1): 143-148.

Gülcin, I.; Oktay, M.; Kirecci, E.; Küfrevioglu, O.I. 2003. Screening of antioxidant and antimicrobial activities of anise (Pimpinella anisun L) seed extracts. Food Chemistry, 83: 371-382.

Madriñán, S. 2004. Rhodostemonodaphne (Lauraceae). Flora Neotropica. Monograph 92. New York, New York Botanical Garden 141, 102 pp.

Mensor, L.L.; Menezes, F.S.; Leitão, G.G.; Reis, A.S.; dos Santos, T.C.; Coube, C.S.; Leitão, S.G. 2001. Screnning of Brazilian plant extracts for antioxidant activity by the use of DPPH free radical method. Phytotherapy Research, 15: 127-130.

Ministério do Meio Ambiente-MMA (Brazilian Ministry of Environment) - Normative Instruction number 6, from September 23, 2008, Annex II: List of Brazilian Flora with deficiency of data.

Miyazama, M.; Watanabe, H.; Umemoto, K.; Kameoka, H. 1998. Inhibition of acetylcholinesterase activity by essential oils of Mentha species. Journal of Agricultural and Food Chemistry, 46 (9): 3431-3434.
Pascual, C.; Gonzalez, R.; Torricella, R. G. 1994. Scavenging action of propolis extract against oxygen radicals. Journal of Ethnopharmacology, 41(2): 9-13.

Passos, G.F.; Fernandes, E.S.; da Cunha, F.M.; Ferreira, J.; Pianowski, L.F.; Campos, M.M.; Calixto, J.B. 2007. Anti-inflammatory and anti-allergic properties of the essential oil and active compounds from Cordia verbenacea. Journal of Ethnopharmacology, 110(2): 323-333.

Peñuelas, J.; Llusià, J. 2002. Linking photorespiration, monoterpenes and hermotolerance in Quercus. NewPhytologist, 155: 227-238.

Peñuelas, J.; Munné-Bosch, S. 2005. Isoprenoids: an evolutionary pool for photoprotection. Trends in Plant Science, 10: 166-169.

Perry, E.K.1986. The cholinergic hypothesis-ten years on. British Medicinal Bulletin, 42: 63-69.

Perry, N.S.L.; Houghtonp, J.; Theobald, A.; Jenner, P.; Perry, E.K. 2000. In-vitro inhibition of human erythrocyte acetylcholinesterase by Salvia lavandulaefolia essential oil and constituents terpenes. Journal of Pharmacy end Pharmacology, 52 (7): 895-902.

Ribeiro, J.E.L.S.; Hopkins, M.J.G.; Vicentini, A.; Sothers, C.A.; Csota, M.A.S.; Brito, J.M.; Souza, M.A.D.; Martins, L.H.P.; Lohmann, L.G.; Assunção, P.A.C.L.; Pereira, E.C.; Silva, C.F.; Mesquita, M.R.; Procopio, L.C. 1999. Flora from Ducke Reserve: Guide of identification of vascular plants of a upland forest Central Amazonia. INPA-DFID: Manaus, AM, Brasil, $816 \mathrm{p}$.

Rhee, I. K.; van de Meent, M.; Ingkaninan, K.; Verpoorte, R. 2001. Screening for acetylcholinesterase inhibitors from Amaryllidaceae using silica gel thin-layer chromarography in combination with bioactivity staining. Journal of Chromatography A, 915: 217-223.

Sacchetti, G.; Maietti, S.; Muzzoli, M.; Scaglianti, M.; Manfredini, S.; Radice, M.; Bruni, R. 2005. Comparative evaluation of 11 essential oils of different origin as functional antioxidants, antiradicals and antimicrobials in foods. Food Chemistry, 91: 621-632.

Telascrea, M.; Araújo, C.C.; Cavalheiro, A.J.; Marques, M.O.M.; Facanali, R., Moraes, P.L.R. 2008. Essential oils from leaves of Cryptocarya spp from the atlantic rain forest. Quimica Nova, 31(3): 503-507.

Zheng, G.Q.; Kenny, P.M.; Lam, L.K.T. 1992. Sesquiterpenes from clove (Eugenia caryphyllata) as potential anticarcinogenic agents. Journal of Natural Products, 55 (7): 999-1003.

Recebido em 02/04/2009

Aceito em 20/01/2010 